\title{
EFEITO DE UM PROTOCOLO DE REPOSIÇÃO HÍDRICA SOBRE O ESTADO DE HIDRATAÇÃO DE ATLETAS APÓS CORRIDA DE DEZ QUILÔMETROS
}

\section{Effect of a fluid replacement protocol on the athletes hydration state after ten kilometers running}

\author{
Julia Teixeira Peixoto de Vasconcellos; Cláudia Mello Meirelles²
}

\author{
${ }^{1}$ NutriCorp Consultoria e Assessoria em Nutrição. \\ ${ }^{2}$ Seção de Pós-Graduação da Escola de Educação Fisica do Exército, Rio de Janeiro - RJ - Brasil. Av. João Luiz Alves s/nº, \\ Urca, Rio de Janeiro - RJ - Brasil. CEP: 22291-090. E-mail: claudiameirelles@yahoo.com.br. Tel: 88747876.
}

\begin{abstract}
Resumo: Objetivo: Determinar a efetividade do protocolo de ingestão de fluidos do American College of Sports Medicine (ACSM, 2007). Métodos: Nove atletas do sexo masculino ( $31 \pm 9$ anos, $76,3 \pm 7,5 \mathrm{~kg}, 1,80 \pm 0,04 \mathrm{~m}$ e $9,8 \pm 5,7 \%$ de gordura corporal) foram submetidos a um teste de corrida de $10 \mathrm{~km}$ em pista de atletismo ao ar livre. Antes e após o teste foram submetidos a medidas de massa corporal (MC); água corporal total (ACT) por impedância bioelétrica; gravidade especifica da urina (GEU) por refratômetro manual e coloração da urina (Ucol) por análise comparativa a uma escala numérica com valores de 1 a 8 correspondendo a possiveis cores da urina. Durante a atividade consumiram água na proporção de $400 \mathrm{~mL} \cdot \mathrm{h}-1$, sendo $90 \mathrm{~mL}$ a cada $10 \mathrm{~min}$. Ao final do teste, a taxa de sudorese (TS) foi calculada. $O$ estudo foi desenvolvido de acordo com as normas éticas estabelecidas pelo Conselho Nacional de Saúde (CNS 196/96). Resultados: A duração média da corrida foi $47 \pm 4$ min e o consumo de água foi $320 \pm 47 \mathrm{~mL}$, o que correspondeu a $410 \pm 48 \mathrm{~mL} \cdot h-1.0$ teste t pareado indicou uma redução de $1,3 \pm 0,2 \%$ na $M C(P=0,000)$ e nenhuma modificação significativa na ACT (de $62,9 \pm 2,8 \mathrm{~L}$ para $63,0 \pm 2,3 \mathrm{~L}$ ), nem na GEU (de $1,014 \pm 0,008$ para $1,015 \pm 0,009$ ). A TS foi de 6,0 $\pm 1,3 \mathrm{~mL} \cdot \mathrm{min}-1$, correspondendo a $358 \pm 79 \mathrm{~mL} \cdot \mathrm{h}-1$. Conclusão: A TS foi inferior ao consumo hídrico e as perdas hidricas não ultrapassaram $2 \%$ da MC, demonstrando que o protocolo de reidratação foi apropriado para manter o estado de hidratação dos atletas.

Palavras-chave: Desidratação, taxa de sudorese, gravidade especifica da urina, protocolo de hidratação.
\end{abstract}

Abstract: Objectives: To determine the effectiveness of the Position Stand on Fluid Replacement from The American College of Sports Medicine Exercise (2007). Methods: Nine male athletes ( $31 \pm 9$ years, $76.3 \pm 7.5 \mathrm{~kg}, 1.80 \pm 0.04 \mathrm{~m} \mathrm{e} 9.8 \pm 5.7 \%$ body fat) were requested to perform a $10 \mathrm{~km}$ run in an outdoor racetrack. Before and after the test, athletes had measured body mass (BM), total body water (TBW) by bioelectric impedance, urine specific gravity (USG) by manual refractometer and urine color (Ucol) by comparison with a numbered scale with values corresponding to colors ranging from 1 to 8 . During the activity they have drunk water in the proportion of $400 \mathrm{~mL} \cdot \mathrm{h}-1$ with a volume of about $90 \mathrm{ml}$ every $10 \mathrm{minutes}$. At the finish of the test, sweating rate (SR) was calculated. This study was developed according to the ethical norms established by the National Health Council from Brazil (CNS 196/96). Results: Athletes' average time on track was $47 \pm 4$ min and the average HR was $168 \pm 9$ b.p.m. The water consumption was $320 \pm 47 \mathrm{~mL}$, corresponding to $410 \pm 48 \mathrm{~mL} \cdot \mathrm{h}-1$. Pared t test showed a reduction of $1.3 \pm 0.2 \%$ in $B M(P=0.000)$ and no significant modifications in TBW (from $62.9 \pm 2.8 \mathrm{~L}$ to $63.0 \pm 2.3 \mathrm{~L}$ ), and USG (from $1.014 \pm 0.008$ to $1.015 \pm 0.009$ ). The $\mathrm{SR}$ was $6.0 \pm 1.3 \mathrm{~mL} \cdot \mathrm{min}-1$, corresponding to $358 \pm 79 \mathrm{~mL} \cdot \mathrm{h}-1$. Conclusion: SR was lower than fluid consumption and the water losses have not exceed $2 \%$ of $B M$, showing that ACSM (2007) recommendation was enough to maintain appropriated water status on this athletes.

Keywords: dehydration, sweating rate, urine specific gravity, hydration protocol

Aceito em 01/04/2011 - Revista de Educação Física 2011 Abr; 151:40-47. Rio de Janeiro - Brasil

\section{INTRODUÇÃO}

O estado de hidratação exerce uma influência crítica no desempenho físico. Embora a hipoidratação pareça não afetar a potência anaeróbia nem a força dinâmica máxima, reduz significativamente a resistência muscular ${ }^{(1)}$ e o desempenho aeróbio durante exercícios de longa duração e alta intensidade, principalmente em ambientes quentes e úmidos ${ }^{(2)}$.

Entre as diversas variáveis que atuam di- retamente sobre as perdas hidricas e eletroliticas se destacam a temperatura do ambiente e a umidade relativa do ar, a intensidade e a duração do exercício, o tamanho e a composição corporal do atleta, o tipo de roupa, os equipamentos utilizados durante a tarefa, entre outros ${ }^{(3,4)}$.

Elevadas taxas de sudorese sem reposição de fluidos podem ocasionar grandes perdas hidricas, as quais podem determinar diferentes graus de desidratação. No entanto, perdas de 
apenas $1 \%$ de massa corporal já são suficientes para elevar a temperatura central ${ }^{(5)}$. A hipertermia, por sua vez, prejudica o desempenho aeróbio por acelerar o distúrbio à homeostase cardiovascular e metabólica ${ }^{(6)}$.

Dependendo da combinação entre as variáveis que determinam as perdas hidricas, pode haver um comprometimento na capacidade de absorção de fluidos pelo trato gastrointestinal. Esta, contudo, pode ser melhorada a partir do hábito de beber líquidos durante o exercício, 0 que favorece a tolerância a maiores volumes de líquidos e reduz a diferença entre perdas e reposição hídrica durante treinos prolongados ${ }^{(3)}$.

O posicionamento do $\operatorname{ACSM}(7)$ recomenda que o consumo de fluidos durante o exercício deve ser suficiente para evitar que as perdas hidricas ultrapassem $2 \%$ da massa corporal. Para tal, aponta que a ingestão de bebidas deve se situar entre $400 \mathrm{~mL}$ a $800 \mathrm{~mL}$ por hora de atividade, considerando que a reposição baseada nos niveis inferiores de recomendação pode ser insuficiente para atletas mais velozes e pesados competindo em ambientes quentes. Contudo, há discussões em paralelo que se opõem a esta recomendação(8).

O efeito de uma sessão de exercícios sobre o estado de hidratação pode ser avaliado por meio de algumas medidas, tais como as alterações do volume plasmático (VP), da coloração da urina (Ucol), da massa corporal (MC), da gravidade específica e osmolaridade da urina (GEU e Uosm, respectivamente) e da água corporal total $(\mathrm{ACT})^{(9)}$.

Sá et al.(10), ao compararem quatro destes indicadores, observaram que as variações na $M C$ e na GEU foram as que demonstraram maior sensibilidade do que o VP e ACT em apontar a desidratação em nove homens submetidos a redução de $2 \%$ na $M C$ por meio de exposição à sauna úmida.

Considerando os efeitos deletérios da desidratação sobre o desempenho, é de suma importância seguir protocolos individuais de hidratação a fim de evitar desidratação excessiva durante o treinamento físico.

O objetivo principal deste estudo foi deter- minar a efetividade do protocolo de ingestão de fluidos recomendado pelo ACSM(7) em evitar que a desidratação ultrapasse $2 \%$ da massa corporal. O objetivo secundário foi comparar os resultados fornecidos por três métodos distintos de avaliação da desidratação de atletas.

\section{MÉTODOS}

\section{Amostra}

Participaram do estudo nove atletas do sexo masculino ( 7 triatletas e 2 corredores), conforme mostra a TABELA 1. Os critérios de exclusão foram o uso de medicamentos ou recursos ergogênicos farmacológicos, tabagismo e a presença de lesões osteomioarticulares. Todos os voluntários preencheram e assinaram um termo de consentimento livre e esclarecido, de acordo com as normas éticas estabelecidas pelo Conselho Nacional de Saúde (Resolução 196/96).

TABELA 1. CARACTERISTICAS DOS ATLETAS.

\begin{tabular}{lccc}
\hline & Média $\pm \mathrm{DP}$ & Minimo & Máximo \\
\hline Idade (anos) & $31 \pm 9$ & 20 & 50 \\
Estatura $(\mathrm{m})$ & $1,80 \pm 0,04$ & 1,74 & 1,85 \\
MC $(\mathrm{kg})$ & $76,3 \pm 7,5$ & 60,7 & 85,8 \\
Gordura (\%) & $11,3 \pm 3,3$ & 7,3 & 17,4 \\
Experiência com corrida (anos) & $2,41,8$ & 1,3 & 7,0 \\
Treinamento semanal (h) & $9,4 \pm 2,9$ & 6,0 & 15,0 \\
\hline
\end{tabular}

DP: desvio padrão; MCT: massa corporal.

\section{Delineamento experimental}

$\mathrm{O}$ estudo constou de duas visitas. Na primeira, os atletas responderam uma anamnese sobre seu treinamento físico semanal e hábitos de hidratação e receberam as orientações necessárias para manter seu nivel de hidratação no dia do teste de corrida. Na segunda tiveram aferidas suas medidas antropométricas e foram submetidos ao teste de corrida. Antes e após o teste foram submetidos a medidas de MC, GEU, ACT e volume urinário após a corrida (VU). Durante a atividade, consumiram água na proporção de $400 \mathrm{~mL}$ por hora e tiveram sua freqüência cardiaca aferida (FIGURA 1). 
GRÁFICO 1: DELINEAMENTO EXPERIMENTAL

$1^{2}$ Visita:

Anamnese, medidas antropométricas, orientaçōes sobre hidratação no dia do teste

\section{$2^{a}$ Visita:}

Teste de comida de $10 \mathrm{~km}$ em pista de atletismo

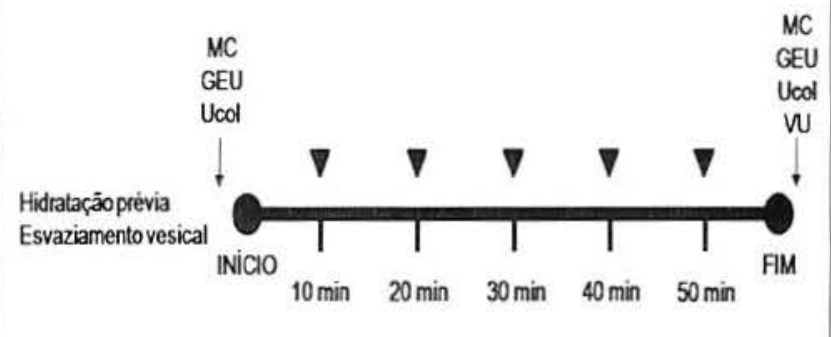

Triångulos negros: ingestäo hidrica; MC: massa corporal; GEU: gravidade especifica da urina; Ucol coloração da urina: VU: volume urinario

Detalhes de obtenção das medidas e equipamentos utilizados

As condições ambientais de temperatura e umidade foram conhecidas por meio de termômetro de bulbo seco e bulbo úmido (Austin, TX, USA). A MC e a ACT foram aferidas em balança com impedância bioelétrica (BIA) bipolar (Tanita BC-553, Tanita Inc, Itabashi-Ku, Tokio, Japão), sendo obtidas após esvaziamento vesical dos sujeitos descalços e trajando sunga de praia. A confiabilidade da estimativa de ACT utilizando o equipamento supracitado demonstrou correlação intraclasse interdias de $R=0,994$ ( $P$ < $0,001)$ e erro típico de $0,23 L(0,78 \%)^{(11)}$.

As dobras peitoral, abdominal e coxa foram utilizadas para estimativa da densidade corporal pela equação de Jackson e Pollock ${ }^{(12)}$ e a porcentagem de gordura corporal pela de Siri(13). A freqüência cardiaca foi monitorada durante a corrida por meio de frequencimetro (Polar S-610, Polar Electro Inc, Kempele, Finlândia).

Para análise da GEU e da Ucol, uma amostra de urina foi coletada antes e após a corrida. A GEU foi determinada a partir da aplicação de uma gota de urina em refratômetro (TS 400, Leica Inc, Wetzlar, Germany) calibrado com água destilada. Para a análise da Ucol, aproximadamente $50 \mathrm{~mL}$ de urina foi comparada por inspeção visual à escala adaptada de Armstrong e recomendada pela National Athletic Trainers' Association(14). Ao final da corrida a urina foi coletada em recipiente próprio graduado em $\mathrm{mi}-$ lilitros, o que permitiu conhecer o VU.

A taxa de sudorese (TS) foi estimada através da equação 1 e expressa em $\mathrm{mL} \cdot \mathrm{min}-1$ :

$T S=\frac{[M C \text { inicial }(\mathrm{kg})+\text { ingestão hidrica }(\mathrm{mL})]-[M C \text { final }(\mathrm{kg})+V U(\mathrm{~mL})]}{\text { tempo }(\mathrm{min})}$ mpo $(\min )$

Onde: $M C=$ massa corporal; $\mathrm{VU}=$ volume urinário após a corrida

Teste de corrida e protocolo de hidratação

$\mathrm{O}$ teste de corrida ocorreu à noite durante o outono e simulou o percurso do triatlo olímpico $(10 \mathrm{~km})$ no menor tempo possivel na pista de atletismo da Fortaleza de São João, situada na cidade do Rio de Janeiro. Os atletas receberam orientações formais de hidratação conforme o posicionamento do $\mathrm{ACSM}^{7}$, ou seja, deveriam ingerir de $5 \mathrm{~mL} \cdot \mathrm{kg}^{-1}$ a $7 \mathrm{~mL} \cdot \mathrm{kg}^{-1}$ de fluidos nas quatro horas anteriores ao teste e $400 \mathrm{~mL} \cdot \mathrm{h}^{-1}$ de água com temperatura entre $15^{\circ} \mathrm{C}$ e $21^{\circ} \mathrm{C}$ durante o teste de corrida.

\section{Tratamento estatístico}

Para comparar os valores de MC, ACT e GEU observados antes e após o teste de corrida foi aplicado um teste t pareado. Para comparar os valores de Ucol aplicou-se o teste de Wilcoxon, por tratar-se de escala intervalar. As alterações percentuais na MC e na TS relativa à MC foram comparadas ao valor de $2 \%$ utilizando um teste $t$ simples para uma amostra (one sample $t$ test), o qual é utilizado para comparar a média de uma variável obtida em estudo com uma média hipotética, geralmente advinda da literatura; neste caso, 2 \% de redução na massa corporal é um ponto de corte que define maiores preocupações com a desidratação. Para comparar os valores de Ucol antes e após o teste de corrida, aplicou-se o teste não paramétrico de Wilcoxon.

A normalidade da distribuição dos valores de cada variável foi testada pelo teste de Shapiro Wilki. Para todas as análises adotou-se um nivel de significância de $P \leq 0,05$ e foi utilizado o software SPSS versão 16.0 (SPSS Inc., Chicago, IL, USA). 


\section{RESULTADOS}

Os testes de corrida ocorreram entre $18 \mathrm{~h}$ e $21 \mathrm{~h}$, com temperatura variando de $21^{\circ} \mathrm{C}$ a 23 ${ }^{\circ} \mathrm{C}$ e umidade relativa do ar entre $63 \%$ e $77 \%$. A duração da corrida foi de $47 \pm 4$ min e a FC média se manteve em $168 \pm 9$ b.p.m. O consumo de água foi $320 \pm 47 \mathrm{~mL}$, o que correspondeu a $410 \pm 48 \mathrm{~mL} \cdot \mathrm{h}^{-1}$.

Embora tenham se hidratado, os atletas reduziram a $M C$ em 1,3 $\pm 0,2 \%(1,0 \pm 0,2 \mathrm{~kg} ; P=$ 0,000 comparado ao valor de $2 \%$ ). ContudoEm adição, o consumo hídrico foi suficiente para garantir que nenhum dos atletas apresentasse desidratação superior a $2 \%$ da MC (FIGURA 2).

A TS média foi de $6,0 \pm 1,3 \mathrm{~mL} \cdot \mathrm{min}^{-1}$, o que correspondeu a $358 \pm 79 \mathrm{~mL} \cdot \mathrm{h}^{-1}$ e ficou abaixo da ingestão hidrica durante a corrida. A FIGURA 2 mostra os valores individuais de TS.

GRÁFICO 2: MODIFICACÕES NA MASSA CORPORAL (A) E TAXA DE SUDORESE (B) DE ATLETAS APÓS CORRIDA DE $10 \mathrm{~km}$

A

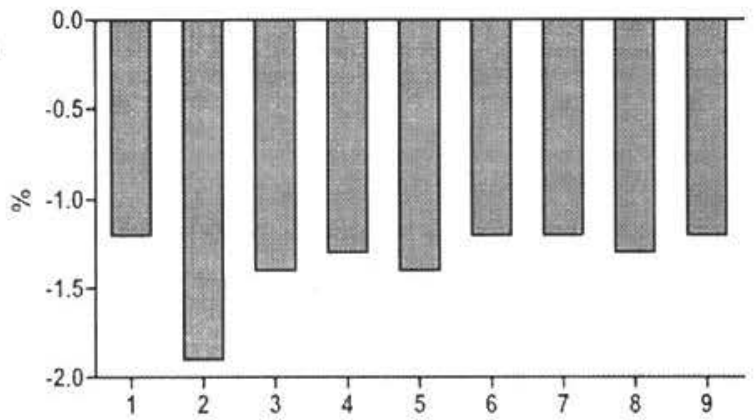

B

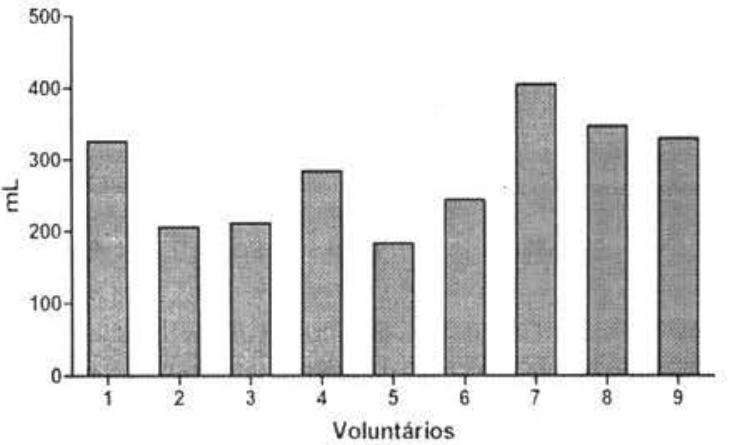

Quantos aos indicadores do estado de hidratação, nenhuma modificação significativa foi observada na ACT (de 62,9 $\pm 2,8 \mathrm{~L}$ para 63,0 $\pm 2,3 \mathrm{~L} ; \mathrm{P}=0,422$ ), na GEU (de 1,014 $\pm 0,008$ para $1,015 \pm 0,009 ; P=0,729)$, nem na Ucol (manteve-se em 3, amplitude 1-4; $P=1,000$ ). A FIGURA 3 mostra o comportamento individual de cada uma destes indicadores.

GRÁFICO 3: COMPORTAMENTO DOS INDICADORES DO ESTADO DE HIDRATAÇÃO DE ATLETAS MEDIANTE CORRIDA DE $10 \mathrm{~km}$.
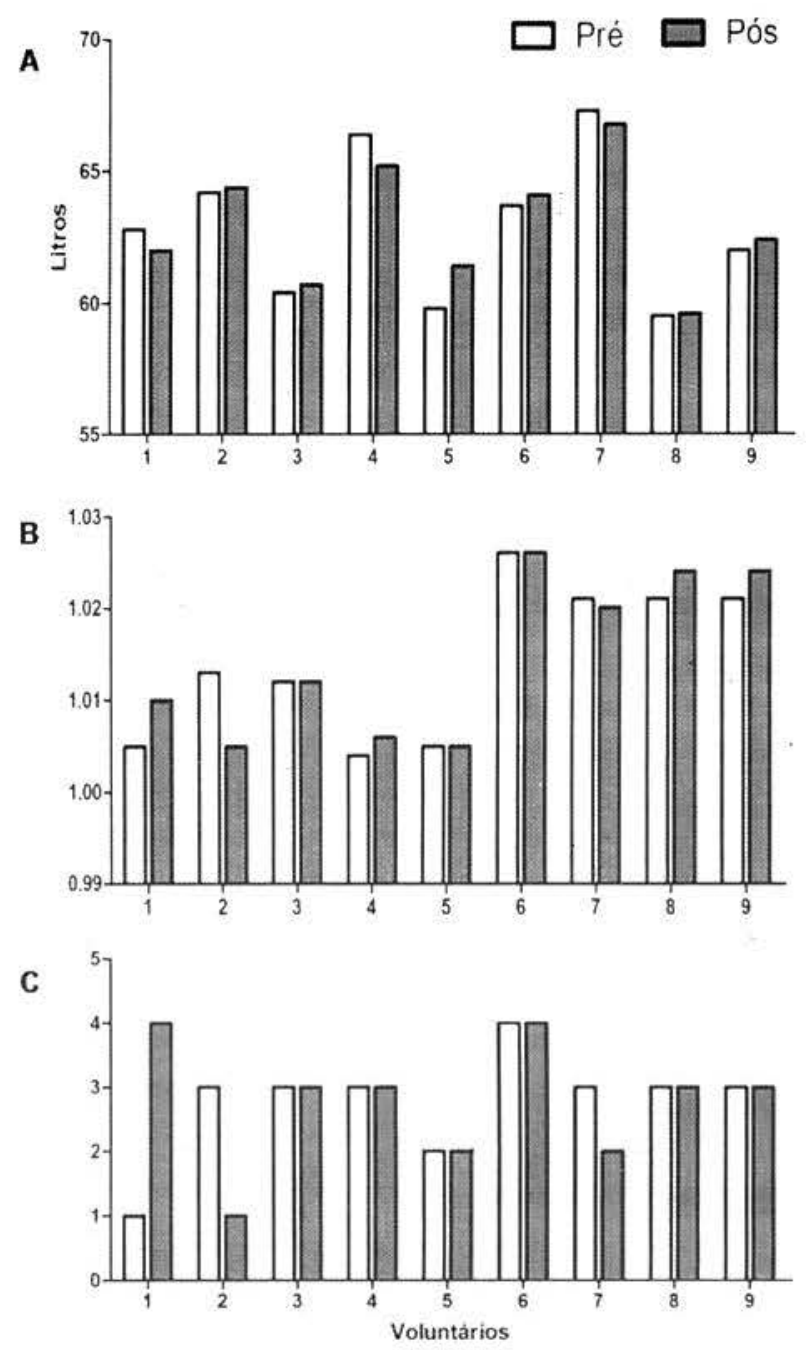

Agua corporal total medida por impedância bioelétrica, B: Gravidade especifica da urina estrmaı por refratômetro, C. Coloraçâo da urina estimada por método comparativo

\section{DISCUSSÃO}

O principal resultado deste estudo sugere que a reposição de fluidos nos valores inferiores do protocolo de reposição do $\operatorname{ACSM}(7)$ foi suficiente para evitar que a desidratação decorrente de uma corrida de $10 \mathrm{~km}$ ao ar livre alcançasse $2 \%$ da massa corporal e a TS superasse a ingestão hídrica.

Já Gomes e Rodrigues ${ }^{(15)}$, que estudaram 12 atletas de uma equipe masculina juvenil de vo- 
leibol de alto nivel, apontaram que a ingestão de $534 \pm 222 \mathrm{~mL} \cdot \mathrm{h}^{-1}$ de água não foi suficiente para compensar a TS de $906 \mathrm{~mL} \pm 276 \mathrm{~mL} \cdot \mathrm{h}^{-1}$, o que resultou em perdas que ultrapassaram $2 \%$ da MC durante um treino típico em ginásio com temperatura média de $22,5 \pm 1,5^{\circ} \mathrm{C}$.

Vasconcelos et al. ${ }^{(16)}$ realizaram estudo de caso com delineamento experimental idêntico ao presente, à exceção de a corrida ter ocorrido ao ar livre durante dias típicos de verão e dos atletas terem sido mais velozes. Observaram que a ingestão hidrica de $400 \mathrm{~mL} \cdot \mathrm{h}^{-1}$ não impediu que as perdas hidricas superassem $2 \%$ da massa corporal em três dos quatro triatletas militares estudados.

Contudo, os achados do presente estudo diferem dos supracitados no que diz respeito à intensidade do exercício físico ${ }^{(15.16)}$, ao protocolo de hidratação prévia ${ }^{(15)}$ e às condições ambientais ${ }^{(16)}$. Em adição, pode-se especular que a diferença de idade e de capacidade aeróbia dos sujeitos podem ter sido fatores que contribuíram para as diferenças na TS nos estudos em questão(17,18).

A evaporação do suor é o principal mecanismo de dissipação de calor durante exercicios intensos em ambiente quente e úmido. Como a sudorese poder ser muito intensa nestas condições, uma adequada ingestão de fluidos pode minimizar a desidratação e reduzir as taxas de aumento da temperatura central ${ }^{(19)}$.

Quando a temperatura ambiente ultrapassa $20^{\circ} \mathrm{C}$, como frequentemente se observa na cidade do Rio de Janeiro, o estresse térmico aumenta e a resposta de fadiga ao exercício físico se instala mais precocemente. Uma das razões relacionadas é a redução mais acelerada dos estoques de energia ocasionada pela desidratação combinada ao estresse térmico.

Alonso et al ${ }^{(20)}$ testaram sete atletas de resistência aeróbia jovens e saudáveis submetidos a exercício até a exaustão (cicloergômetro a $\sim 61$ $\%$ do $\mathrm{VO}_{2}$ máx) em duas condições: desidratação progressiva e reposição hídrica. Foram observadas taxas de depleção do glicogênio muscular significativamente maiores nos atletas na primeira comparada à segunda condição $(298 \pm 21 \mathrm{mmol} /$ $\mathrm{kg}-1$ vs $205 \pm 15 \mathrm{mmol} / \mathrm{kg}-1$ respectivamente).
A literatura comumente defende que a reposição hidrica em volumes equivalentes às perdas pela sudorese pode prevenir o declínio no débito cardiaco por meio da manutenção da volemia e do volume de ejeção ventricular, beneficiando, desta forma, a termorregulação, pois aumenta o fluxo sanguineo periférico, facilitando a transferência de calor interno para a periferia(19).

Recentes argumentos vão de encontro a estas ideias e abrem uma nova discussão baseada em algumas premissas, a saber, (a) a carência de evidências que mostrem que repor tanta água quanto perdida pelo suor é melhor do que o consumo ad libitum; (b) os diversos excelentes resultados em competições internacionais obtidos por atletas que pouco se hidrataram durante suas provas; (c) o exagero na ingestão hidrica pode ocasionar hiponatremia em atletas submetidos a atividades intensas e prolongadas no calor e (d) a existência de um possivel conflito de interesses quando pesquisadores recebem fomento da indústria de bebidas hidroeletrolíticas. Assim, Timothy Noakes, um pesquisador da Universidade da Cidade do Cabo, na África do Sul defende que a reposição de fluidos durante atividades prolongadas deve ocorrer sim, mas precisa obedecer apenas a sede do atleta, ou seja, ser ad libitum, não devendo obedecer qualquer protocolo pré-estabelecido de reidratação. Contudo, uma discussão mais aprofundada sobre este assunto foge ao escopo do presente estudo e pode ser lida em outras publicações ${ }^{(8,21-24)}$.

Quanto aos métodos de avaliação do estado de hidratação, os resultados apontaram que a monitoração da MC, considerando-se sua simplicidade foi um método útil para detectar as modificações no estado de hidratação decorrentes da corrida de $10 \mathrm{~km}$.

No presente estudo, a BIA não se mostrou adequada à identificação das alterações na ACT, concordando com estudos anteriores ${ }^{(10.25-27)}$ que reportaram que o método não foi útil na monitoração de modificações na ACT mediante desidratação por meio de sauna ou exercício. Assim, embora a BIA seja um método acessivel, rápido, não-invasivo, confiável e válido para a estimativa da ACT e avaliação da composição corporal|(28), os 
resultados disponiveis na literatura indicam que em situação nas quais a desidratação é acompanhada por alterações abruptas na osmolaridade plasmática e nas concentrações de eletrólitos, a $\mathrm{BIA}$ perde confiabilidade e validade para estimar a ACT.

Alterações significativas na MC mediante desidratação aguda podem estar desacompanhadas também de modificações significativas na GEU. Sá et al. ${ }^{\left({ }^{10)}\right)}$ relataram que desidratação de $\sim 2 \%$ da $\mathrm{MC}$ por meio de sauna úmida alteraram a GEU de $1,016 \pm 0,008$ para $1,025 \pm 0,006(P>0.05)$. Popowski et al. ${ }^{(29)}$ também não encontraram alterações significativas na GEU (de 1,009 $\pm 0,006$ para $1,007 \pm 0,005$ ) após desidratação de $1 \%$ da MC por meio de sauna seca e exercício em cicloergômetro. Parece que apenas em situações de desidratação mais severa - a partir de $3 \%$ da $M C$ - as alterações na GEU são passiveis de ser detectadas por refratômetro(29).

Corroborando o exposto acima, Pinheiro et al. $^{(30)}$ apontaram que a GEU foi um indicador sensivel de desidratação de 59 militares jovens submetidos a um teste de marcha de $20 \mathrm{~km}$. No estudo foi estimada uma perda hidrica média de $4100 \mathrm{~mL}$ durante o percurso, o que representaria aproximadamente $5,7 \%$ da massa corporal média dos sujeitos.

De acordo com Shirreffs ${ }^{(9)}$, a literatura não dispõe de um padrão-ouro para identificação do estado de hidratação, mas aponta que índices urinários são marcadores úteis. No entanto, em se tratando de identificar modificações agudas decorrentes de periodos de rápido turnover hidrico - como é o caso do presente estudo - parece que a medida da osmolaridade do plasma seria a mais sensivel, por apresentar relação linear com o estado de hidratação. Contudo, a coleta de urina tem a vantagem de não ser invasiva e de não depender de técnica e equipamentos rebuscados quando comparada a métodos que dependem de coleta de sangue, a qual ainda tem o agravante de poder ser estressante para alguns indivíduos.

Para utilização das medidas urinárias é necessário considerar que existem limitações na interpretação de seus resultados. A GEU pode ser influenciada pela presença de solutos como uréia, glicose e proteínas. Já a Ucol pode ser influenciada por uma série de fatores que não possuem relação com o estado de hidratação, incluindo alimentos, medicamentos e a presença de doenças(9). No presente estudo, na tentativa de reduzir tais interferências, os voluntários deveriam ser saudáveis e não fazer uso de medicamentos, suplementos ou alimentos nas duas horas que antecederiam o teste da corrida.

No entanto, mesmo tomados estes cuidados, a GEU e a Ucol não se apresentaram como bons indicadores das alterações agudas do estado de hidratação dos atletas do presente estudo, conforme apontado na revisão de literatura de Shirreffs $(9)$.

\section{CONCLUSÃO}

Os resultados do presente estudo apontaram que a ingestão de $400 \mathrm{~mL}$ de água - valor inferior da recomendação de reposição de fluidos do $\operatorname{ACSM}(7)$ - foi adequada para evitar que a desidratação alcançasse $2 \%$ da massa corporal após corrida de $10 \mathrm{~km}$, ao menos nos atletas e condições presentes no dia de teste. Em adição, concluiu-se que a medida da massa corporal apresentou-se como o método útil para identificar as perdas hídricas agudas, quando comparada à monitoração da água corporal total, gravidade especifica e coloração da urina, sugerindo que este método rápido, simples e não-invasivo possa ser empregado quando da não disponibilidade ou necessidade de métodos mais rigorosos e invasivos.

Para interpretação das informações do presente estudo, é importante se observar que este apresenta algumas limitações, tais como a não utilização de equipamentos voltados à monitoração mais acurada das variáveis fisiológicas relacionadas ao objeto de estudo, o número reduzido da amostra, a incerteza do cumprimento do protocolo de hidratação pré-corrida pelos sujeitos, o fato de o teste de corrida ter sido realizado à noite, ou seja, em temperatura mais amena do que naturalmente se encontraria durante horários matutinos ou vespertinos e, finalmente, as variações climáticas inter-testagens, uma vez que não foi possivel submeter todos os atletas ao teste em 
um único dia. Desta forma, tornam-se necessária a realização de estudos que considerem e transponham tais limitações, a fim de contribuir com o avanço do conhecimento.

\section{REFERÊNCIAS BIBLIOGRÁFICAS}

1. Sá CK, Meirelles CM, Mazza R, Gomes PSC. Effects of acute dehydration and rehydration on muscle strength, power, and endurance of trained males. Med Sci Sports Exerc 2005;37(5):S264 [abstract].

2. Cheuvront, S. N., R. Carter, and M. N. Sawka. Fluid balance and endurance performance. Curr. Sports Med. Rep. 2:202-208, 2003.

3. Rehrer NJ. Fluid and electrolyte balance in ultra-endurance sport. Sports Med 2001; 31(10): 701-15.

4. Naghii MR. The significance of water in sport and weight control. Nutr Health. 2000; 14(2): 127-32.

5. Sawka MN, Montain SJ, Latzka WA. Hydration effects on thermoregulation and performance in heat. Comp Bioch Physiol Part A 2001;128:679-90.

6. Gonzalez-Alonso J, Calbet JAL. Reductions in systemic and skeletal muscle blood flow and oxygen delivery limit maximal aerobic capacity in humans. Circulation 2003; 107; 824-30.

7. American College of Sports Medicine (ACSM). Position Stand on exercise and fluid replacement. Med Sci Sports Exerc 2007:377-90.

8. Noakes TD. Drinking guidelines for exercise: What evidence is there that athletes should drink "as much as tolerable", "to replace the weight lost during exercise" or "ad libitum"? J Sports Sci 2007;25(7):781-96.

9. Shirreffs SM. Markers of hydration status. Eur J Clin Nutr 2003;57(S2):S6-S9.

10. Sá CK, Meirelles CM, Mazza R, Gomes PSC. Determinação da sensibilidade de indicadores do estado de hidratação de atletas. Rev Bras Fisiol Exerc 2004;3(1):106 [resumo].

11. Vasconcelos JTP, Meirelles CM, Confiabilidade das medidas de água corporal total e gordura corporal percentual pela impedância bioelétrica. Rev Ed Fis 2007;139:100 [resumo].
12. Jackson AS, Pollock ML.Generalized equations for predicting body density of men. Br J Nutr 1978;40(3):497504.

13. Siri WE. Body Composition from fluid spaces and density. Em J Brozek e A Henschel (Eds.). Technics for Measuring Body Composition. 1961; p. 223-4. Washington DC: National Academy of Sciences.

14. Casa DJ, Armstrong LE, Hillman SK, Montain SJ, Reiff RV, Rich BSE, Roberts WO, Stone JA. National Athletic Trainers' Association Position Statement: fluid replacement for athletes. J Athl Train 2000;35(2):212-4.

15. Gomes ACV, Rodrigues LOC, Avaliação do estado de hidratação dos atletas, estresse térmico do ambiente e custo calórico do exercício durante sessões de treinamento em voleibol de alto nivel. Rev Paul Educ Fís 2001; 15(2): 201-11.

16. Vasconcellos J, Labanca J, Guerra I. Aplicação de um protocolo de hidratação à corrida de $10 \mathrm{~km}$ com equipe de triatlo do exército brasileiro. Anais do $31^{\circ} \mathrm{Sim}-$ pósio Internacional de Ciências do Esporte 2008;186.

17. Gant N, Williams C, King J, Hodge BJ. Thermoregulatory responses to exercise: relative versus absolute intensity. J Sports Sci 2004;22(11-12):1083-90.

18. Kondo N, Takano S, Aoki K, Shibasaki M, Tominaga $\mathrm{H}$, Inoue $\mathrm{Y}$. Regional differences in the effect of exercise intensity on thermoregulatory sweating and cutaneous vasodilation. Acta Physiol Scand 1998;164(1):71-8.

19. American College of Sports Medicine (ACSM). Position Stand on exertional heat illness during training and competition. Med Sci Sports Exerc 2007:557-72.

20. Alonso JG, Calbet JAL, Bodil N. Metabolic and thermodynamic responses to dehydration-induced reductions in muscle blood flow in exercising humans. Journal of Physiology. 1999; 520(2): 577-589.

21. Noakes TD. Hydration in the marathon using thirst to gauge safe fluid replacement. Sports Med 2007;37(45):463-6.

22. Fudge BW, Easton C, Kingsmore D, Kiplamai FK, Onywera VO, Westerterp KR et al. Elite Kenyan endurance runners are hydrated day-to-day with ad libitum fluid intake. Med Sci Sports Exerc 2008; 40(6):1171-9.

23. Beltrami FG, Hew-Butler T, Noakes TD. Drinking policies and exercise-associated hyponatraemia: is anyone still promoting overdrinking? $\mathrm{Br} \mathrm{J}$ Sports Med 2008;42(10):496-501. 
24. Noakes T. Fluid replacement during marathon running. Clin J Sport Med 2003;13(5):309-18.

25. Koulmann N, Jimenez C, Regal D, Bolliet $P$, Launay JC, Savourey $G$ et al. Use of bioelectrical impedance analysis to estimate body fluid compartments after acute variations of the body hydration level. Med Sci Sports Exerc 2000;32(4):857-64.

26. Berneis $\mathrm{K}$, Keller U. Bioelectrical impedance analysis during acute changes of extracellular osmolality in man. Clin Nutr. 2000;19(5):361-6.

27. Pialoux V, Mischler I, Mounier R, Gachon P, Ritz P, Coudert J, et al. Effect of equilibrated hydration changes on total body water estimates by bioelectrical impedance analysis. Br J Nutr 2004;91(1):153-9.

28. Mattsson S, Thomas BJ. Development of methods for body composition studies. Phys Med Biol 2006;51:R203-8.
29. Popowski, LA, Oppliger, RA, Lambert,GP, Johnson, RF, Johnson, AK, Gisolfi,CV. Blood and urinary measures of hydration status during progressive acute dehydration. Med Sci Sports Exerc 2001; 33 (5) 747 753.

30. Pinheiro LRL, Abreu RSL, Kroeff MB, Barbosa ES, Santos Jr DMS, Gomes FC et al. Modificação nos indicadores do estado de hidratação de candidatos ao curso de Ações de Comando após marcha de $20 \mathrm{Km}$. Rev Ed Fis 2006;134:41-7.

Endereço para correspondência:

Cláudia de Mello Meirelles.

Seção de Pós-Graduação da Escola de Educação Fisica do Exército - Av. João Luiz Alves, s/n ${ }^{\circ}$ - Urca

Rio de Janeiro - RJ - Brasil. CEP: 22291-090

E-mail: claudiameirelles@yahoo.com.br 